\title{
Una proposta (o una sfida?) per chirurghi vascolari
}

\section{Tony Talluri}

\author{
Akern R\&D, Firenze
}

\section{L'} cronico è forse uno dei maggiori problemi tuttora irrisolti. Mi sono posto alla ricerca di possibili soluzioni in seguito a problemi cardiovascolari che mi hanno forzato al trattamento cronico di ldl-aferesi. Le mie povere vene sono costrette ad accettare almeno una volta al mese un ago il cui calibro è quasi identico a quello del vaso, per estrarre almeno 100 millilitri al minuto.

Ora capisco cosa deve sopportare un paziente in dialisi che viene trattato con frequenza quasi giornaliera e con flussi ancor più elevati!

Le vene sono vasi "normalmente vuoti" e le arterie sono vasi "normalmente pieni". Questa differenza dettata dalla loro differente funzione (trasporto del sangue da e per il cuore) li rende profondamente diversi anche dal punto di vista strutturale. Tuttavia la necessità di poter trattare in circolazione extracorporea volumi ematici significativi hanno portato ad accettare la congiunzione chirurgica di un'arteria con una vena, e quindi un controsenso fisiologico che a lungo andare non può non creare problemi.

La professoressa Luisa Berardinelli che ammiro e ritengo uno dei migliori chirurghi vascolari mi è testimone di diversi tentativi, più o meno intelligenti, ma dobbiamo ammettere che la strada delle soluzioni "artificiali" (leggi protesi o simili) è piena di difficoltà. Lo scoglio principale è che pur disponendo di materiali sempre più biocompatibili, non e possibile rispettare la fisiologia.
L'amico Marco Lombardi mi ha recentemente stimolato con la sua ottima idea di creare uno spazio letterario, con approccio multidisciplinare in grado di dare voce a varie competenze. Il taglio della rivista mi ha finalmente sbloccato nel lanciare un'idea tenuta nel cassetto da diverso tempo, che per quanto pazza possa apparire la reputo realizzabile.

In pratica la proposta è quella di copiare un sistema naturale, che funzio-

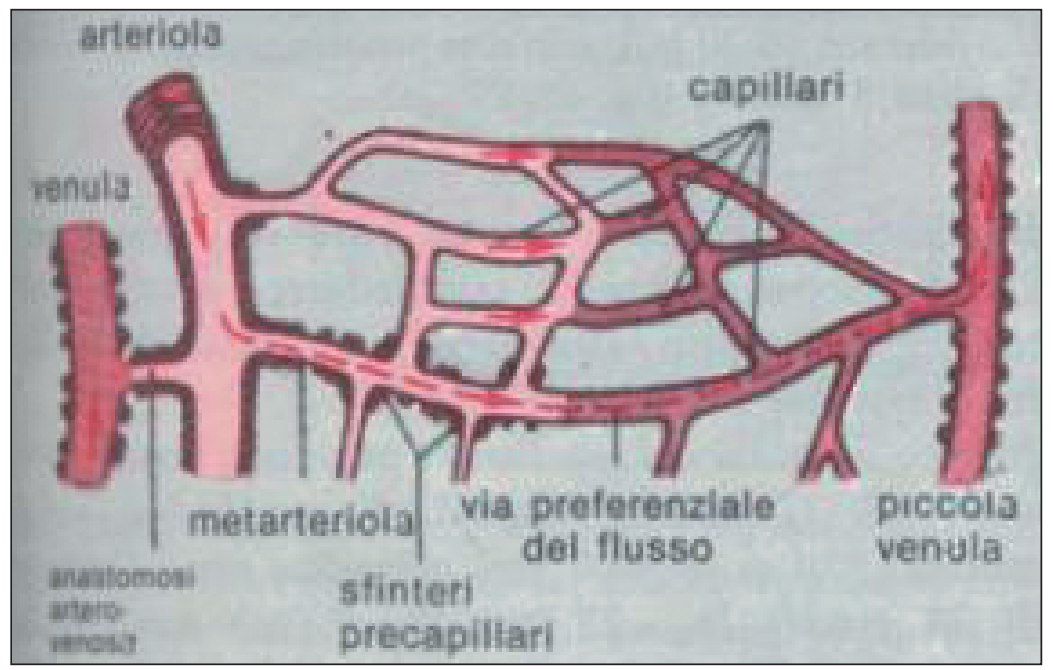




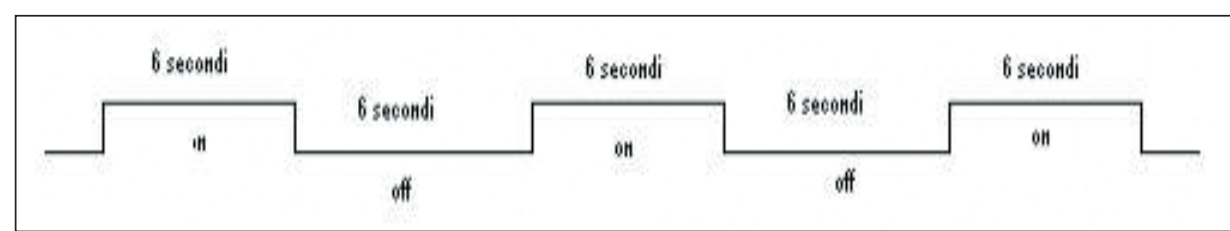

Fig. 2

na perfettamente e realizzarne uno similare, su scala più grande. Lo scambio ematico fra venule e arteriole nel microcircolo permette di modulare rapidamente ed efficacemente gli scambi artero-venosi, tramite dei piccoli sfinteri. Ritengo possibile ricostruire per via chirurgica un sistema simile, unendo una vena e un'arteria con un condotto di unione dotato di "sfintere". Il controllo dello scambio artero-venoso è ottenibile attraverso un muscolo che chirurgicamente viene avvolto al condotto la cui contrazione è modulabile attraverso una semplice elettrostimolazione. Se non ricordo male negli anni Ottanta una strada similare è stata attuata per controllare l'incontinenza rettale attraverso la rilocazione del muscolo gracilis. La Figura 1 mostra schematicamente l'anastomosi fra venule e arteriole da replicare in larga scala con una vena e un'arteria.

Un sistema similare potrebbe essere ricostruito chirurgicamente. La congiunzione fra l'arteria e la vena prescelte deve avvenire con una tecnica di anastomosi ampia, che permette di lasciare un tratto idoneo a essere avvolto da un fascio muscolare situato in prossimità di tali vasi, per poterlo "strozzare" quando stimolato elettricamente.

Considerando che l'arresto completo del flusso o la sua parzializzazione continua possono essere fibrinogeni, con conseguente "clotting" del vaso, si sfrutta il fenomeno negativo della fatica muscolare indotta dalla stimolazione elettrica. Il rilasciamento del muscolo, necessario al suo riposo è favorevole per il lavaggio del vaso.

Lo stimolatore elettrico avrà almeno 2 elettrodi attivi impiantati in modo idoneo a reclutare le fibre muscolari che saranno attivate alternativamente con un "duty cycle" del 50\%, come da schema della Figura 2.
Un ciclo di lavoro simile è in grado di ridurre il flusso arterioriso del $50 \%$.

È tuttavia possibile aumentare ulteriormente la fase "on" fino a ridurre il flusso del $75 \%$, senza incontrare fatica muscolare.

Lo stimolatore elettrico è facilmente realizzabile con una tecnica identica ai comuni pacemaker e impiantato in una sacca.

È chiaro che durante la fase di trattamento con circolazione extracorporea, lo stimolatore deve essere disattivato per permettere il flusso continuo. L'accensione e lo spegnimento sono facilmente attuabili, come nei pacemakers, attraverso un comando in RF trasmesso da un'antenna, mentre il "duty cicle" è residente come programma nello stimolatore stesso.

\section{akern@akern.com}

\section{BIBLIOGRAFIA}

Cavina E, Seccia M, Evangelista G. A new technique for ileo and colostomy continence. Preliminary report on development of experimental electro-stimulated and the relative surgical solution: neostomy and neo-sfincter. M.C. Rivista di Medicina e Chirurgia 1979; 1-4: 47-56.

Cavina E, Seccia M, Evangelista G. Una nuova tecnica per la continenza delle ileocolostomie. A new technique for ileo and colostomy continence. M.C. Rivista di Medicina e Chirurgia 1980; 1:31-58.

Cavina E, Menconi M, Balestri R, Seccia M. Static-dynamic graciloplasty: a reconstructive technique of the anal sphincter after abdominoperineal resection of the rectum. Meeting 92nd Convention ASCRS - Chicago, 2-7 May 1993. Abstr Book Dis Colon Rectum 1993; 36 (4): 29. 